\title{
Capacidade funcional de idosos atendidos em unidades básicas de saúde do SUS
}

\author{
Functional capacity of elderly patients attended in SUS primary healthcare units \\ Capacidad funcional de ancianos atendidos en unidades básicas de salud del SUS
}

\begin{abstract}
Calíope Pilger', Mario Umberto Menon", Thais Aidar de Freitas Mathias"I
'Universidade de São Paulo, Escola de Enfermagem de Ribeirão Preto, Programa de Pós-Graduação em Enfermagem (Doutoranda). Ribeirão Preto-SP, Brasil.

"Universidade Estadual do Centro-Oeste, Centro de Ciências Agrárias e Ambientais. Irati-PR, Brasil.

I" Universidade Estadual de Maringá, Centro de Ciências da Saúde, Departamento de Enfermagem. Maringá-PR, Brasil.
\end{abstract}

\section{Submissão: 15-06-2012 Aprovação: 06-11-2013}

\section{RESUMO}

O estudo objetivou analisar a capacidade funcional dos idosos cadastrados nas Unidades Básicas de Saúde do município de Guarapuava-PR, Brasil. Participaram 359 idosos, entrevistados de janeiro a abril de 2010, utilizando-se a seção I e IV do questionário Brazil Old Age Schedule. Os resultados demonstraram que 89,9\% apresentaram algum grau de dependência, dos quais $70,8 \%$ apresentaram dependência leve e 19,2\% moderada ou grave. As mulheres possuem maior grau de dependência leve $(70,5 \%)$ e moderada e grave $(62,3 \%)$. Os fatores associados com o grau de dependência entre idosos residentes em Guarapuava foram faixa etária, grau de escolaridade, arranjo familiar, trabalho remunerado, local de residência, estado conjugal e renda familiar. Conclui-se que a maior parte dos idosos na comunidade é acometida por limitações e convive com algum tipo de dependência para a realização de atividades básicas da vida diária.

Descritores: Perfil de Saúde; Avaliação da Capacidade de Trabalho; Saúde do Idoso; Enfermagem.

\section{ABSTRACT}

This study aimed to analyze the functional capacity of elderly registered in Basic Health Units of Guarapuava-PR, Brazil. The subjects were 359 elderly, interviewed from January to April 2010, using Section I and IV of the questionnaire Brazil Old Age Schedule. The results showed that $89.9 \%$ had some degree of dependency, of which $70.8 \%$ light and $19.2 \%$ moderate or severe dependence. Women have a higher degree of mild $(70.5 \%)$ and moderate and severe dependence $(62.3 \%)$. Factors associated with the degree of dependence among the elderly living in Guarapuava were age, schooling, living arrangements, employment, place of residence, marital status and family income. It is concluded that most of the elderly in the community is affected by limitations and live with some kind of dependence to perform basic activities of daily living.

Key words: Health Profile; Work Capacity Evaluation; Health of the Elderly; Nursing.

\section{RESUMÉN}

El estudio objetivó analizar la capacidad funcional de los ancianos inscritos en Unidades Básicas de Salud de la GuarapuavaPR, Brasil. Participaran 359 personas mayores, entrevistadas entre enero y abril de 2010, con la Sección I y IV del cuestionario Brazil Old Age Schedule. Los resultados mostraron que 89,9\% tenían algún grado de dependencia, de los cuales 70,8\% tenían una dependencia leve y $19,2 \%$ moderada o grave. Las mujeres tenían un mayor grado de dependencia leve $(70,5 \%)$ y moderada y grave $(62,3 \%)$. Los factores asociados con el grado de dependencia entre los ancianos que viven en Guarapuava fueron la edad, la escolaridad, arreglos de vivienda, empleo, lugar de residencia, estado civil y el ingreso familiar. Se concluye que la mayoría de las personas mayores en la comunidad se ve afectada por las limitaciones y vivir con algún tipo de adicción para realizar actividades básicas de la vida diaria.

Palabras clave: Perfil de Salud; Evaluación de Capacidad de Trabajo; Salud del Anciano; Enfermería.

\section{AUTOR CORRESPONDENTE Calíope Pilger E-mail: caliopepilger@hotmail.com}




\section{INTRODUÇÃO}

O acelerado ritmo do crescimento da população idosa é observado mundialmente, incluindo o Brasil e outros países latino-americanos. A expectativa de vida dos brasileiros que em 1900 não alcançava os 35 anos de idade, em 1950 atingiu 43 anos, em 200068 anos e existe a expectativa de atingir os 80 anos em $2025^{(1)}$.

Enquanto reduz-se a mortalidade por doenças infecto-contagiosas, cresce a prevalência de doenças crônicas não transmissíveis (DCNT) em consequência do aumento da longevidade o que por sua vez podem levar a incapacidade funcional tanto na vida adulta como também após os 60 anos de idade ${ }^{(2)}$.

A capacidade funcional pode ser definida como o potencial que uma pessoa apresenta para decidir e atuar de forma independente em sua vida, no seu cotidiano ${ }^{(1)}$. Embora o conceito de capacidade funcional seja complexo abrangendo outros conceitos como os de deficiência, incapacidade, desvantagem, bem como os de autonomia e independência, na prática trabalha-se com o conceito de capacidade ou incapacidade. Define-se incapacidade funcional pela presença de dificuldade no desempenho de certos gestos e de certas atividades da vida cotidiana ou mesmo pela impossibilidade de desempenhá-las ${ }^{(3)}$.

Os fatores mais fortemente associados com a capacidade funcional estão relacionados com a presença de algumas doenças, deficiências ou problemas médicos. Entretanto, observa-se, que a principal hipótese subjacente em alguns dos estudos que identificam as associações é a de que, além dos fatores biológicos e a presença de doenças crônicas, a capacidade funcional é influenciada por fatores demográficos, socioeconômicos, culturais e psicossociais. Desse modo, são citados como potenciais fatores explicativos da capacidade funcional alguns comportamentos relacionados ao estilo de vida como fumar, beber, comer excessivamente, não realizar exercícios, padecer de estresse psicossocial agudo ou crônico e não manter relações sociais e de apoio $^{(3)}$.

Por isso, estudos sobre a capacidade funcional em idosos tornam-se necessários, primeiro para o entendimento de como as pessoas estão vivendo os anos adicionais ganhos com o aumento da longevidade e assim subsidiar as atividades e ações de saúde desenvolvidas em serviços de saúde na atenção primária. A capacidade funcional é mais um indicador da consequência de um processo de doença do que uma medida de incapacidade ou de morbidade específica. A capacidade funcional vem se tornando um conceito particularmente útil para avaliar o estado de saúde dos idosos na comunidade, porque muitos convivem com várias doenças simultaneamente, que variam em severidade e causam diferentes impactos na vida cotidiana ${ }^{(4)}$.

A perda da capacidade funcional está associada à predisposição de fragilidade, dependência, institucionalização, risco aumentado de quedas, morte e problemas de mobilidade, trazendo complicações ao longo do tempo, e gerando cuidados de longa permanência e alto custo(5).

Assim, este estudo teve como objetivo analisar a capacidade funcional dos idosos cadastrados nas Unidades Básicas de Saúde do município de Guarapuava - PR, Brasil.

\section{MÉTODO}

Estudo transversal, integrante de um estudo mais abrangente em amostra representativa dos idosos cadastrados nas Unidades Básicas de Saúde residentes no município de Guarapuava, estado do Paraná, Brasil, com inquérito domiciliar, que analisou suas condições sociodemográficas e de saúde.

Guarapuava, com população estimada, em 2009, de 172.128 habitantes e 14.981 idosos é sede da $5^{\text {a }}$ Regional de Saúde do Estado, com coeficiente de mortalidade infantil, em 2009 , de 18,8 por mil nascidos vivos e taxa de mortalidade materna de 83,4 por 100.000 nascidos $^{(6)}$. Quanto à oferta de serviços de saúde o município conta com 30 Unidades Básicas de Saúde (UBS) distribuídas em 10 distritos sanitários, com 29 Equipes de Saúde da Família (ESF). Cada Distrito Sanitário conta com um Centro Integrado de Atendimento (CIA) que são unidades básicas de saúde de atendimento à população, que se diferenciam das ESF por apresentarem especialidades médicas, como ginecologia e obstetrícia e pediatria além de serviços de odontologia ${ }^{(7)}$.

A ficha A, um dos instrumentos de cadastro da família na Estratégia Saúde da Família- ESF, foi utilizada para levantamento dos idosos cadastrados e para a elaboração da amostra do estudo. A relação das pessoas com 60 anos e mais de idade, constando o nome e endereço, foi cedida pelas enfermeiras responsáveis por cada uma das UBS. A partir do total de idosos foi realizado o cálculo da amostra por meio de amostragem estratificada proporcional ao número de idosos em cada UBS como segue:

$$
\mathrm{n} 0=\frac{\left(\mathrm{z}_{\alpha} / 2\right)^{2}}{4 \mathrm{~d}^{2}}
$$

Assim, o número de idosos a ser entrevistados, estabelecido para a pesquisa, foi de 359. Foi realizado um sorteio por meio da técnica de amostragem aleatória simples para selecionar os idosos que estavam descritos em ordem numérica crescente nas listas das UBS.

Para as entrevistas foi utilizado o questionário Brazil Old Age Schedule (BOAS) ${ }^{(8)}$ um instrumento que avalia a vida do idoso de uma forma multidimensional, composto por nove seções. Foram utilizadas para este estudo as seções I (dados sociodemográficos), II (percepção de saúde) e IV (capacidade funcional). As entrevistas foram realizadas de janeiro a abril de 2010 .

A capacidade funcional dos idosos foi avaliada por meio da escala de atividades da vida diária (AVD) presentes no questionário BOAS, composta por 15 atividades: banhar- se, vestir-se, ir ao banheiro em tempo, deitar, levantar-se da cama ou cadeira, alimentar-se sozinho, pentear-se, cortar as unhas dos pés, subir um lance de escada, andar no plano (atividades básicas para a manutenção corporal ou atividades pessoais AVDP), preparar refeições, subir escadas, pegar ônibus, ir andando a um lugar perto de sua casa, tomar remédios na hora certa e fazer limpeza de casa (atividades básicas para o convívio independente na comunidade ou instrumentais - AVDI). O grau de dependência do idoso foi classificado segundo: ausência de dependência (não apresenta incapacidade ou 
dificuldade em nenhuma das AVD), dependência leve (incapacidade ou dificuldade para realizar de uma a três das AVD) e dependência moderada e/ou grave (incapacidade ou dificuldade em realizar quatro ou mais AVD) ${ }^{(8)}$.

A análise dos fatores associados ao grau de dependência foi observada segundo variáveis sociodemográficas: idade (60 a 69,70 a 79 e 80 anos ou mais); sexo; escolaridade (nenhuma, primário, ginásio ou $1^{\circ}$ grau, $2^{\circ}$ grau completo, curso superior); arranjo familiar (mora sozinho, mora com alguém); trabaIho remunerado (aposentado, aposentado e trabalho remunerado); local de residência (zona rural e zona urbana); situação conjugal (casado ou morando junto, viúvo, divorciado, nunca se casou ou solteiro) e de percepção de saúde que é entendida como aspecto subjetivo e particular dos indivíduos. A análise de associação foi feita por meio de estatística indutiva ou inferencial, com a realização do teste de qui-quadrado (chi-square - $X^{2}$ ), teste de Fisher utilizando o software Estatistic.

As entrevistas foram realizadas no domicílio dos idosos, de janeiro a abril de 2010, por entrevistadores devidamente treinados. As entrevistas foram aplicadas aos idosos ou também ao seu cuidador, quando o idoso tivesse alguma limitação em relação à comunicação. Foram registradas 13 recusas. As entrevistas foram realizadas após o consentimento dos entrevistados e assinatura do termo de consentimento livre e esclarecido (TCLE).
O Projeto foi aprovado pelo Comitê Permanente de Ética em Pesquisa Envolvendo Seres Humanos da Universidade Estadual de Maringá-PR conforme a Resolução 196/96 do Conselho Nacional de Saúde (Parecer 492/2009).

\section{RESULTADOS}

As mulheres referiram mais dependência funcional que os homens, tanto na dependência leve $(77,5 \%$ contra $58,6 \%)$ quanto na dependência moderada ou grave $(33,6 \%$ contra $20,3 \%$ ). Os idosos de 60 a 69 anos e de 70 a 79 anos referiram mais frequentemente dependência leve do que os idosos com mais de 80 anos. Por outro lado, a dependência moderada ou grave foi referida por $81,6 \%$ dos idosos de 70 a 79 anos e por $89,5 \%$ dos idosos com 80 anos ou mais.

Nota-se que entre 70 e 79 anos a prevalência de dependência moderada/grave é 3 (três) vezes maior quando comparada à faixa de 60 a 69 anos e 6 vezes quando comparada à 80 anos ou mais de idade. A capacidade funcional moderada ou grave está mais presente nas mulheres com $\mathrm{RP}=2,9 \%$ o está associado à dependência, pois apresentou nível de significância de $\mathrm{p}=0,0002$. Ainda, aumenta 3 (vezes) a prevalência das mulheres possuem dependência moderada (Tabela 1).

Tabela 1 - Idosos segundo capacidade funcional e variáveis sociodemográficas. Guarapuava-PR, Brasil, 2010.

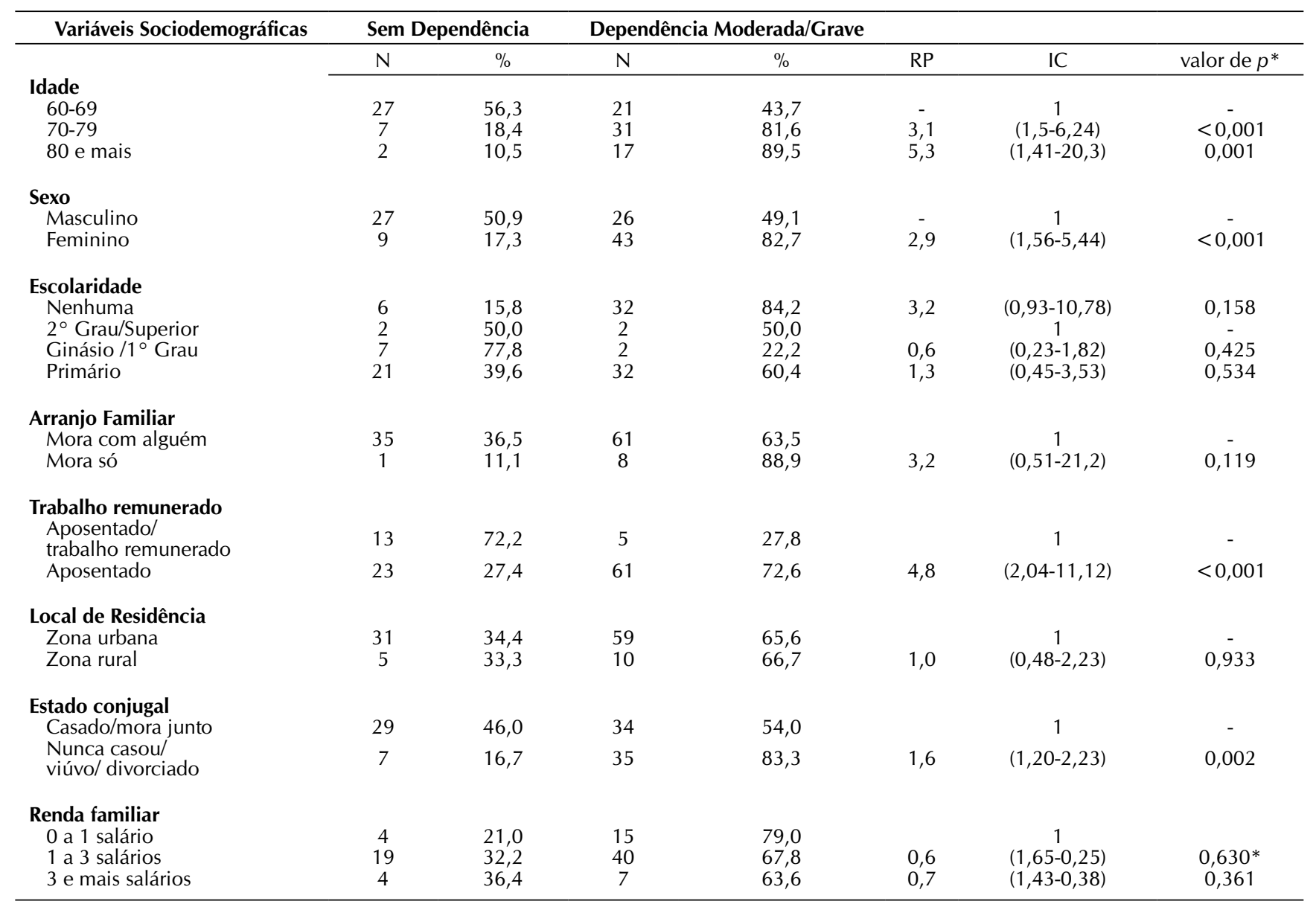

*Teste de Fisher ** Percentuais calculados excluindo-se as variáveis sem resposta ou ignoradas. 
Observou-se que, quanto menor o grau de escolaridade, maior a prevalência de dependência moderada/grave, aumentando 1,2 para o nível primário e 3,1 para nenhuma escolaridade e não sabe ler e escrever. A dependência moderada/ grave apresentou uma prevalência de 3,2 vezes maior para idosos que moram sozinhos quando comparada a morar com familiares. Para viúvos, solteiros e os que nunca se casaram a prevalência de dependência moderada/grave foi de 1,6.

Similar a dependência moderada/grave, o sexo feminino esta fortemente associada a ter dependência leve, com razão de prevalência de $2,5(p<0,001)$.

Os idosos que não sabem ler e escrever e moram só estão associados a dependência leve ( $R P=5,1, p=0,0249)$. Morar só está associado a dependência leve ( $R P=1,2)$ (Tabela 3). Ser viúvo, divorciado ou nunca casou está associado tanto a dependência leve como moderada/grave.

Observa-se na Tabela 3 a prevalência alta de idosos com dependência leve que considera sua saúde ótima ou boa ( $p=$ $0,010)$. A prevalência de quedas foi similar tanto no idoso sem dependência como com dependência leve.

Com relação ao grau de dependência moderada/grave observa-se que $44,9 \%$ dos idosos perceberam sua saúde como ruim e $15,9 \%$ como péssima ( $p=0,0000$ e $p=0,0010)$. Na percepção da acuidade visual $23,2 \%$ dos idosos com dependência moderada/grave perceberam péssima $(R P=1,1$ e $p=$ 0,235) (Tabela 04).

Tabela 2 - Capacidade funcional de idosos sem dependência e com dependência leve segundo variáveis sociodemográficas. Guarapuava-PR, Brasil, 2010.

\begin{tabular}{|c|c|c|c|c|c|c|c|}
\hline \multirow[t]{2}{*}{ Variáveis Sociodemográficas } & \multicolumn{2}{|c|}{ Sem Dependência } & \multicolumn{2}{|c|}{ Dependência Leve } & \multirow[b]{2}{*}{$\mathrm{RP}$} & \multirow[b]{2}{*}{ IC } & \multirow[b]{2}{*}{ Valor de $p$} \\
\hline & $\mathrm{N}$ & $\%$ & $\mathrm{~N}$ & $\%$ & & & \\
\hline \multicolumn{8}{|l|}{ Idade } \\
\hline $60-69$ & 27 & 13,8 & 169 & 86,2 & & 1 & - \\
\hline $70-79$ & 7 & 9,9 & 64 & 90,1 & 0,8 & $(0,92-0,74)$ & $<0,001$ \\
\hline 80 e mais & 2 & 8,7 & 21 & 91,3 & 1,0 & $(0,92-1,19)$ & 0,496 \\
\hline \multicolumn{8}{|l|}{ Sexo } \\
\hline Masculino & 27 & 26,5 & 75 & 73,5 & & 1 & - \\
\hline Feminino & 9 & 4,8 & 179 & 95,2 & 2,5 & $(1,80-3,57)$ & $<0,001$ \\
\hline \multicolumn{8}{|l|}{ Escolaridade } \\
\hline $\begin{array}{l}\text { Curso } \\
\text { Superior / } 2^{\circ} \mathrm{Grau}\end{array}$ & 2 & 28,6 & 5 & 71,4 & & 1 & - \\
\hline Ginásio ou $1^{\circ} \mathrm{Grau}$ & 7 & 41,2 & 10 & 58,8 & 0,7 & $(2,63-0,17)$ & $0,562^{*}$ \\
\hline Primário & 21 & 13,0 & 141 & 87,0 & 2,5 & $(0,54-11,95)$ & 0,238 \\
\hline Nenhuma / não sabe ler e escrever & 6 & 5,8 & 97 & 94,2 & 5,1 & $(1,23-21,18)$ & $0,025^{*}$ \\
\hline \multicolumn{8}{|l|}{ Arranjo Familiar } \\
\hline Mora com alguém & 35 & 14,0 & 214 & 86,0 & & 1 & - \\
\hline Mora só & 1 & 2,4 & 40 & 97,6 & 1,2 & $(1,01-1,32)$ & $0,036^{*}$ \\
\hline \multicolumn{8}{|l|}{ Trabalho remunerado } \\
\hline Aposentado e trabalho remunerado & 13 & 23,2 & 43 & 76,8 & & 1 & - \\
\hline Aposentado & 23 & 10,0 & 208 & 90,0 & 2,1 & $(1,22-3,65)$ & 0,007 \\
\hline \multicolumn{8}{|l|}{ Local de residência } \\
\hline Zona urbana & 31 & 14,8 & 179 & 85,2 & & 1 & - \\
\hline Zona rural & 5 & 6,2 & 75 & 93,8 & 1,2 & $(1,00-1,49)$ & $0,049 *$ \\
\hline \multicolumn{8}{|l|}{ Estado conjugal } \\
\hline Casado/morando Junto & 29 & 16,8 & 144 & 83,2 & & 1 & \\
\hline Nunca casou / viúvo / divorciado & 7 & 6,0 & 110 & 94,0 & 1,4 & $(1,10-1,83)$ & $0,006^{*}$ \\
\hline \multicolumn{8}{|l|}{ Renda familiar } \\
\hline 0 a $1 *$ salário & 4 & 4,6 & 83 & 95,4 & & 1 & \\
\hline 1 a 3 salários & 19 & 13,6 & 120 & 86,4 & 0,4 & $(0,91-0,20)$ & $0,028^{*}$ \\
\hline 3 e mais salários & 4 & 20,0 & 16 & 80,0 & 0,6 & $(0,92-0,39)$ & $0,018^{*}$ \\
\hline
\end{tabular}

* Teste exato de Fisher** Percentuais calculados excluindo-se as variáveis sem resposta ou ignoradas. 
Tabela 3 - Capacidade funcional de idosos sem dependência e com dependência leve segundo percepção de saúde. Guarapuava-PR, Brasil, 2010.

\begin{tabular}{|c|c|c|c|c|c|c|c|}
\hline \multirow[t]{2}{*}{ Percepção de Saúde } & \multicolumn{2}{|c|}{ Sem Dependência } & \multicolumn{2}{|c|}{ Dependência Leve } & \multirow[b]{2}{*}{$\mathrm{RC}$} & \multirow[b]{2}{*}{ IC } & \multirow[b]{2}{*}{ Valor de $p$} \\
\hline & $\mathrm{N}$ & $\%$ & $\mathrm{~N}$ & $\%$ & & & \\
\hline \multicolumn{8}{|l|}{ Percepção de Saúde } \\
\hline Ótima/Boa & 31 & 86,1 & 160 & 63,0 & 1,0 & & - \\
\hline Ruim & 4 & 11,1 & 78 & 30,7 & 1,3 & $1,07-1,63$ & 0,0101 \\
\hline Péssima & - & - & 15 & 5,9 & 1,1 & $0,99-1,21$ & 0,0904 \\
\hline \multicolumn{8}{|l|}{ Percepção Acuidade Visual } \\
\hline Ótima/Boa & 23 & 63,9 & 134 & 52,8 & 1,0 & & - \\
\hline Ruim & 12 & 33,3 & 100 & 39,4 & 1,1 & $0,86-1,53$ & 0,4496 \\
\hline Péssima & 1 & 2,8 & 20 & 7,9 & 1,1 & $0,95-1,28$ & 0,2435 \\
\hline \multicolumn{8}{|l|}{ Percepção Acuidade Auditiva } \\
\hline Ótima/Boa & 25 & 69,4 & 193 & 76,0 & 1,0 & & - \\
\hline Ruim & 11 & 30,6 & 56 & 22,0 & 0,9 & $1,10-0,73$ & 0,2861 \\
\hline Péssima & - & - & 4 & 1,6 & 1,0 & $0,97-1,08$ & 0,4721 \\
\hline \multicolumn{8}{|l|}{ Teve quedas } \\
\hline Sim & 5 & 13,9 & 40 & 15,7 & 1,0 & & - \\
\hline Não & 31 & 86,1 & 213 & 83,9 & 0,9 & $2,06-0,37$ & 0,7660 \\
\hline \multicolumn{8}{|l|}{ Problemas de Saúde } \\
\hline Sim & 18 & 50 & 120 & 47,2 & 1,0 & & - \\
\hline Não & 18 & 50 & 133 & 52,4 & 1,1 & $0,74-1,51$ & 0,7727 \\
\hline \multicolumn{8}{|c|}{ Internação nos últimos 3 meses } \\
\hline Sim & 4 & 11,1 & 15 & 5,9 & 1,0 & & - \\
\hline Não & 32 & 88,9 & 238 & 93,7 & 1,9 & $0,66-5,35$ & 0,2404 \\
\hline \multicolumn{8}{|l|}{ Toma remédio } \\
\hline Sim & 23 & 63,9 & 195 & 76,8 & 1,0 & & - \\
\hline Não & 13 & 36,1 & 59 & 23,2 & 0,8 & $1,03-0,67$ & 0,0940 \\
\hline
\end{tabular}

* Percentual calculado excluindo-se as variáveis sem resposta ou ignoradas.

Tabela 4 - Capacidade funcional de idosos sem dependência e com dependência moderada/grave, segundo percepção de saúde. Guarapuava-PR, Brasil, 2010.

\begin{tabular}{|c|c|c|c|c|c|c|c|}
\hline \multirow[t]{2}{*}{ Percepção de Saúde } & \multicolumn{2}{|c|}{ Sem Dependência } & \multicolumn{5}{|c|}{ Dependência Moderada/Grave } \\
\hline & $\mathrm{N}$ & $\%$ & $\mathrm{~N}$ & $\%$ & RC & IC & Valor de $\mathrm{p}$ \\
\hline \multicolumn{8}{|l|}{ Percepção de Saúde } \\
\hline Ótima/Boa & 31 & 86,1 & 27 & 39,1 & 1,0 & & - \\
\hline Ruim & 4 & 11,1 & 31 & 44,9 & 1,9 & $1,39-2,60$ & 0,0000 \\
\hline Péssima & - & - & 11 & 15,9 & 1,4 & $1,15-1,73$ & 0,0010 \\
\hline \multicolumn{8}{|l|}{ Percepção Acuidade Visual } \\
\hline Ótima/Boa & 23 & 63,9 & 24 & 34,8 & 1,0 & & - \\
\hline Ruim & 12 & 33,3 & 29 & 42,0 & 1,5 & $0,98-2,14$ & 0,0600 \\
\hline Péssima & 1 & 2,8 & 16 & 23,2 & 1,6 & $1,19-2,14$ & 0,0016 \\
\hline \multicolumn{8}{|l|}{ Percepção Acuidade Auditiva } \\
\hline Ótima/Boa & 25 & 69,4 & 42 & 60,9 & 1,0 & & - \\
\hline Ruim & 11 & 30,6 & 23 & 33,3 & 1,1 & $0,52-2,98$ & 0,6228 \\
\hline Péssima & - & - & 4 & 5,8 & 1,1 & $0,97-1,23$ & 0,1290 \\
\hline \multicolumn{8}{|l|}{ Teve quedas } \\
\hline Sim & 5 & 13,9 & 13 & 18,8 & 1,0 & & - \\
\hline Não & 31 & 86,1 & 55 & 79,7 & 0,7 & $1,85-0,29$ & 0,5025 \\
\hline \multicolumn{8}{|l|}{ Problemas de Saúde } \\
\hline Sim & 18 & 50 & 39 & 56,5 & 1,0 & & - \\
\hline Não & 18 & 50 & 29 & 42,0 & 0,9 & $1,27-0,60$ & 0,4735 \\
\hline \multicolumn{8}{|l|}{ Internacão nos últimos 3 meses } \\
\hline Sim & 4 & 11,1 & 11 & 15,9 & 1,0 & & - \\
\hline Não & 32 & 88,9 & 58 & 84,1 & 0,7 & $2,00-0,24$ & 0,5019 \\
\hline \multicolumn{8}{|l|}{ Toma remédio } \\
\hline Sim & 23 & 63,9 & 55 & 79,7 & 1,0 & & - \\
\hline Não & 13 & 36,1 & 14 & 20,3 & 0,8 & $1,03-0,63$ & 0,0782 \\
\hline
\end{tabular}

*Percentuais calculados excluindo-se as variáveis sem resposta ou ignoradas 
Ao analisar os idosos que possuem dependência leve e dependência moderada/grave e que utilizam remédios, pôde-se observar que a prevalência é similar, 76,8\% e 79,7\%, respectivamente.

\section{DISCUSSÃO}

A incapacidade funcional ou desabilidade é processo dinâmico e progressivo, consequência das DCNT e de mudanças fisiológicas associadas ao processo de envelhecimento podendo ocorrer de forma aguda, como por exemplo, no acidente vascular encefálico e na fratura de fêmur, que ocasionam limitações funcionais. Denominam-se limitações funcionais as restrições na realização de ações físicas e operações mentais fundamentais para a vida diária, em comparação às pessoas de mesmo sexo e faixa etária. Portanto, incapacidade funcional ou desabilidade é a limitação para a realização das atividades de vida diária AVD, comprometendo a capacidade funcional do indivíduo para manter-se independente ${ }^{(9)}$.

Na população de idosos, a incapacidade funcional é um dos indicadores mais comuns usados como preditor de saúde e é utilizado especialmente para avaliar as necessidades sociais e de utilização dos serviços de saúde ${ }^{(10)}$. A capacidade funcional abrange dois grupos de atividades: atividades de vida diária (AVD) e atividades instrumentais de vida diária (AIVD). Esse indicador tem sido estudado para avaliar a performance dos idosos nas atividades básicas e instrumentais de vida diária, que são considerados determinantes e fundamentais da independência pessoal e da capacidade dos indivíduos de manter uma interação social, de papéis e de trabalho(10).

No presente estudo, quando avaliado o grau de dependência segundo a gravidade, 70,8\% dos idosos apresentaram incapacidade leve, e 19,2\% apresentaram incapacidade moderada ou grave. A incapacidade leve representa um fator para a incapacidade $^{(11)}$. A incapacidade funcional é um fenômeno que difere entre homens e mulheres. A prevalência de incapacidade para a realização de AVD foi significativamente mais alta entre as mulheres em comparação aos homens. Existem algumas hipóteses que explicam essa diferença, como a maior sobrevivência das mulheres em relação aos homens; a maior prevalência de condições incapacitantes não fatais entre as mulheres (osteoporose, osteoartrite e depressão, por exemplo) e a maior habilidade da mulher para reportar maior número de condições de saúde em relação aos homens da mesma faixa etária(11).

A idade mostrou-se um fator fortemente associado à diminuição da capacidade funcional. Os idosos com 80 anos ou mais tiveram alta prevalência de algum grau de incapacidade funcional, quando comparados aos de 60-69 anos o que concorda com resultados de outros estudos, como o de Joaçaba - SP para o qual idosos de 70 anos ou mais apresentam positivamente associadas com capacidade funcional adequada ${ }^{(1)}$. Estudo realizado em Belo Horizonte - MG demonstrou que entre idosos, o risco de incapacidade funcional dobra a cada década de vida. A dificuldade para realizar as AVD aumentou progressivamente com a idade ${ }^{(11)}$.

Com relação à variável arranjo familiar, na análise dos fatores para dependência moderada/grave foi observado que morar só está associado com incapacidade funcional. Em um estudo multicêntrico conduzido na Finlândia, Holanda e Espanha, que comparou a prevalência, a incidência e a recuperação da incapacidade entre idosos que vivem na comunidade, foi demonstrado que, mesmo quando existem diferenças culturais, os laços sociais (familiares e não familiares) são protetores da incapacidade na velhice ${ }^{(12)}$. Quando considerada a incidência da incapacidade, os laços familiares têm maiores efeitos protetores do que os laços não familiares, mas quando considerada a prevalência da incapacidade, os laços não-familiares são mais protetores ${ }^{(11)}$.

A capacidade funcional pode ser influenciada por fatores sociodemográficos, econômicos, culturais, psicossociais, como comportamentos relativos ao estilo de vida, como fumar, beber, comer excessivamente, manter relações sociais, conjugais, dentre outros. Sabe-se que o estado de viuvez pode influenciar negativamente a capacidade funcional do idoso ${ }^{(3)}$, o que pode sugerir os resultados do presente estudo que mostraram associação significativa entre a viuvez e dependência grave ou moderada.

Dos fatores associados à diminuição na capacidade funcional, alguns podem ser revertidos ou minimizados. A baixa situação econômica, a falta de segurança que sentem em relação ao pequeno poder aquisitivo de que dispõem aumentam as chances de perda de autonomia. Esse fator pode estar ligado também à forma como o idoso se sente no momento, isto é, após a aposentadoria. Alguns idosos passam a viver com rendas menores, e este fator pode influenciar sua autoestima, comunicação, convívio social, com familiares, amigos, pois com a aposentadoria o idoso pode relacionar à perda de sua função na sociedade, na família e do seu estado funcional ${ }^{(1)}$.

A prevalência de utilização de serviços públicos de saúde por $71 \%$ dos idosos com dependência moderada ou grave pode indicar a necessidade de cuidados à saúde, além da importância de criação de estratégias que garantam o acesso à assistência médica na atenção básica a estes indivíduos ${ }^{(13)}$.

A associação de quedas com dependência leve e modera$\mathrm{da} /$ grave e remete ao fato das quedas serem de natureza multifatorial e dependerem de muitas condições, como o uso de drogas, nível de atenção de cuidadores, familiares e do próprio idoso, além da prevalência e severidade de $\mathrm{DCNT}^{(14)}$. Isso pode explicar porque idosos deste estudo obtiveram prevalência de quedas similares ao apresentar tanto a dependência leve como a moderada/grave.

Os dados deste estudo sobre o grau de dependência dos idosos de Guarapuava, um município de médio porte no sul do Brasil sugerem que essa população tem sido acometida por limitações no seu cotidiano, convivendo com graus variados de dependência na realização de atividades simples. Essa realidade reforça a importância de se conhecer a capacidade funcional do idoso e transformar esta atividade parte integrante da avaliação clínica realizada pela equipe de saúde e de enfermagem.

De acordo com esta realidade, os profissionais de saúde também necessitam realizar ações de promoção e prevenção que devem iniciar no domicílio por meio da higiene, ventilação, alimentação, imunizações e detecção precoce de doenças, pois, quando a pessoa está doente ou acamada, é possível 
prevenir a piora do quadro, evitando-se imobilizações e perda de funcionalidade ${ }^{(15)}$.

As limitações funcionais podem ser consideradas um processo que precede as condições de incapacidade. Identificar os fatores sociodemográficos, de saúde que podem estar relacionados com as incapacidades funcionais auxilia na elaboração de políticas públicas com objetivo de preservar a independência dos idosos ${ }^{(16)}$.

\section{CONSIDERAÇÕES FINAIS}

Observa-se a importância da realização de estudos epidemiológicos de base domiciliar, fundamentais para conhecer a distribuição dos fatores de risco presentes na população além de levantar um perfil da situação socioeconômica, de saúde, com fins de auxiliar na implementação de ações e políticas de saúde para as pessoas com mais de 60 anos de idade.

Os resultados sobre a capacidade funcional sugerem que a maior parte dos idosos entrevistados tem sido acometida por limitações no seu cotidiano, convivendo com dependência, principalmente de grau leve, na realização de atividades simples, reforçando a importância de avaliar a capacidade funcional do idoso e transformar esta atividade uma parte integrante da avaliação de enfermagem e da equipe de saúde na atenção básica.

No ponto de vista da Enfermagem, ressalta-se a importância do enfermeiro frente à sua atuação na saúde do idoso, em especial na atenção básica, pois, conhecendo o idoso na comunidade, é possível considerá-lo em suas múltiplas interfaces, o que é de extrema importância para subsidiar a gestão do cuidado a essa clientela de maneira a preservar a sua independência e autonomia, além de promover melhor bem estar físico, psíquico e social.

\section{REFERÊNCIAS}

1. Fiedler MM, Peresv KG. Capacidade funcional e fatores associados em idosos do Sul do Brasil: um estudo de base populacional. Cad Saúde Pública. 2008;24(2):409-415.

2. Benedetti TRB, Gonçalves LHT, Mota JAPS. Uma proposta de política de atividade física para idosos. Texto \& Contexto Enferm. 2007;16(3):287-398.

3. Rosa TEC, Benicío MHD, Latorre MRDO, Ramos LR. Fatores determinantes da capacidade funcional entre idosos. Rev Saúde Pública. 2003;37(1):40-8.

4. Parahyba MI, Veras R, Melzer D. Incapacidade funcional entre as mulheres idosas no Brasil. Rev Saúde Pública. 2005;39(3):383-91.

5. Reis LA, Torres GV. Influência da dor crônica na capacidade funcional de idosos institucionalizados. Rev Bras Enferm. 2011;64(2):274-80

6. SESA. Departamento de Vigilância epidemiológica DEVE. Divisão de informações epidemiológicas- DVIEP. Sistemas de Informações sobre nascidos vivos - SINASC. Sistemas de informações sobre mortalidade -SIM 2009. Acesso em: 28 set 2009. Disponível em: http://www. saude.pr.gov.br/arquivos/File/boletim_epid/2009/Boletim_Epidemiologico_N30.pdf.

7. Ministério da Saúde [homepage na internet]. Caderno de Informações de Saúde. [acesso em 03 nov 2009]. Disponível em: http://tabnet.datasus.gov.br/tabdata/cadernos/ pr.htm.

8. Veras R, Dutra S. Perfil do idoso brasileiro: questionário BOAS [monografia na internet]. Rio de Janeiro: UERJ, UnATI; 2008 [acesso em 08 jul 2009]. Disponível em: http://www.crde-unati.uerj.br/liv_pdf/perfil.pdf.

9. Bornardi G, Azevedo e Souza VB, Moraes JFD. Incapacidade funcional em idosos: um desafio para os profissionais de saúde. Sci Med. 2007;17(3):138-144.

10. Arias-Merino ED, Mendoza-Ruvalcaba NM, Ortiz GG, Velázquez-Brizuela IE, Meda-Lara RM, Cueva-Contreras J. Physical function and associated factors in communitydwelling elderly people in Jalisco, Mexico. Arch Gerontol Geriatr. 2012;(54):271-278

11. Giacomin KC, Peixoto SV, Uchoa E, Lima-Costa MF. Estudo de base populacional dos fatores associados à incapacidade funcional entre idosos na Região Metropolitana de Belo Horizonte, Minas Gerais, Brasil. Cad Saúde Pública. 2008;24(6):1260-1270.

12. Zunzunegui MV, Rodriguez-Laso A, Otero A, Pluijm SMF, Nikula S, Blumstein T. et al. Disability and social ties: comparative findings of the CLESA study. Eur J Ageing. 2005;2:40-7.

13. Rodrigues MAP, Facchini LA, Piccini LX, Tomasi E, Thumé E, et al. Uso de serviços básicos de saúde por idosos portadores de condições crônicas, Brasil. Rev Saúde Pública. 2009;43(4):604-12.

14. Kato-Narita EM, Nitrini R, Radanovic M. Assessment of balance in mild and moderate stages of Alzheimer's disease: implications on falls and functional capacity. Arq Neuropsiquiatr. 2011;69(2-A):202-207.

15. Meireles VC, Matsuda LM, Coimbra JAH, Mathias TAF. Características dos idosos em área de abrangência do Programa Saúde da Família na Região Noroeste do Paraná: contribuições para a gestão do cuidado em enfermagem. Saúde Soc. 2007;16(1):69-80.

16. Torres GV, Reis LA, Reis LA. Assessment of functional capacity in elderly residents of an outlying area in the hinterland of Bahia/Northeast Brazil. Arq Neuropsiquiatr. 2010;68(1):39-43. 